\title{
INCLUSÃO E INFLUÊNCIA DE CARACTERÍSTICAS ESPACIAIS EM MODELOS DE REGULAÇÃO FLORESTAL
}

\author{
Daniel Henrique Breda Binoti ${ }^{*}$, Mayra Luiza Marques da Silva Binoti², Helio Garcia Leite ${ }^{1}$, \\ José Marinaldo Gleriani ${ }^{1}$, Carlos Antonio Álvares Soares Ribeiro ${ }^{1}$
}

*Autor para correspondência: danielhbbinoti@gmail.com

RESUMO: Neste estudo, objetivou-se propor formas alternativas de inclusão de restrições ambientais sobre os modelos de regulação florestal. Os modelos tiveram como função objetivo: i) a minimização das variações das distâncias entre as unidades de gestão com a intervenção da colheita e da unidade onde a produção é entregue, ii) a maximização do valor presente líquido (VPL), com a aplicação da variação permitida em distância (de 5, 10, 15 e 20\%) entre as unidades de gestão com a intervenção da colheita da unidade onde a produção é entregue; iii) maximizar a distância entre as unidades de gestão com a assistência da colheita; iv) minimização da variação da distância entre as unidades de gestão, com intervenções de colheita; v) a maximização do valor presente líquido (VPL), com a aplicação da variação permitida em distância (de 5, 10, 15 e 20\%) entre as unidades de gestão com a intervenção da colheita. Os modelos foram avaliados economicamente pelo valor presente líquido (VPL) e ambientalmente pelo Indice de área de corte (IAC). As formulações propostas apresentaram-se ambientalmente superiories a formulação clássica (Modelo I).

Palavras-chave: Restrições de adjacência, regulação florestal, algoritmos genéticos.

\section{INCLUSION AND EFFECT OF SPATIAL CHARACTERISTICS ON MODELS OF FOREST REGULATION}

\begin{abstract}
The objective of this study was to propose alternative ways of including environmental constraints on models of forest regulation. The propose were minimization of the variations of the distances between the management units with harvest intervention and the unit where production is delivered; maximization of the net present value (NPV), with the application of allowed variation in distance (of 5, 10, 15 and 20\%) between the management units with harvest intervention the unit where production is delivered; maximizing the distance between management units with assistance from harvesting; minimization of the variation of the distance between the management units with harvest interventions; maximization of the net present value (NPV), with the application of allowed variation in distance (of 5, 10, 15 and $20 \%$ ) among the management units with harvest intervention. The models were evaluated economically by the net present value (NPV) and environmentally by a harvest area index (IAC). The proposed formulations were environmentally better than the classical formulation (Model I).
\end{abstract}

Key words: Adjacency restrictions, forest regulation, genetic algorithm.

\section{INTRODUÇÃO}

A inclusão de aspectos espaciais nos modelos de regulação florestal é importante quando características ambientais e sociais são consideradas. No planejamento florestal espacial, a localização e as interações entre as unidades de manejo (UM) são integradas no modelo de regulação e a disposição das UM é considerada durante a otimização (HEINONEN, 2007).

A realização de atividades silviculturais em uma unidade de manejo pode influenciar as unidades de manejo adjacentes, sendo que esta influência decresce drasticamente com a distância (STRANGE et al., 2002). Por exemplo, a colheita de uma unidade de manejo pode expor os talhões vizinhos a danos causados por vento, problemas de drenagem e erosão (BASKENT; KELES, 2005; CHURCH et al., 1998).
Dentre os objetivos para a inclusão de restrições espaciais nos modelos de regulação pode-se destacar a redução da abertura de extensas áreas de corte, a manutenção de corredores ecológicos entre fragmentos de floresta nativa, a redução do assoreamento de rios, a proteção da fauna e o manejo da paisagem (BASKENT; KELES, 2005).

Quando características espaciais são incluídas nos modelos de regulação, as variáveis que representam as unidades de manejo deixam de ser contínuas e passam a ser discretas, cada unidade de manejo deve ser então, assinalada a apenas uma prescrição. A programação inteira (PI) e a programação inteira mista (PIM) são utilizadas para a resolução desse tipo de problema (HILLER; LIEBERMAN, 2010). Contudo, problemas reais podem apresentar enormes dimensões e alta complexidade

${ }^{1}$ Universidade Federal de Viçosa - Viçosa, Minas Gerais, Brasil

${ }^{2}$ Universidade Federal dos Vales do Jequitinhonha e Mucuri - Diamantina, Minas Gerais, Brasil

Cerne, Lavras, v. 20, n. 1, p. 157-164, jan./mar. 2014 
(BETTINGER et al., 1999; MURRAY; CHURCH, 1995). Os algoritmos tradicionais de programação matemática são ineficientes na resolução desses problemas sendo necessária a utilização de técnicas heurísticas (AARTS; LENSTRA, 1997; BARE; MENDOZA, 1992; BASKENT; JORDAN, 1991, 2002; BETTINGER et al., 2007; RODRIGUES et al., 2003, 2004a, 2004b; WANG; ZHANG, 2001).

A utilização de técnicas heurísticas possibilita como vantagem a incorporação de características não lineares na formulação das restrições e da função objetivo do modelo de regulação. Quando as heurísticas são utilizadas para a solução de modelos multi-objetivos, o componente espacial pode ser incluído na função objetivo, utilizando funções de penalidade (BASKENT; JORDAN, 2002; ÖHMAN; ERIKSSON, 2002).

As meta-heurísticas mais utilizadas em modelos de regulação são simulated annealing (BASKENT; JORDAN, 2002; LOCKWOOD; MOORE, 1993; RODRIGUES, 2004), tabu search (BETTINGER et al., 1997; RICHARDS; GUNN, 2003; RODRIGUES, 2001) e algoritmos genéticos (BETTINGER et al., 2002; PUKKALA; KURTTILA, 2005; RODRIGUES, 2001). Uma significativa contribuição sobre o emprego de heurísticas, para a resolução de modelos de regulação da produção de florestas equianêas, é decorrente dos trabalhos realizados por Binoti (2010), Rodrigues (2001) e Rodrigues (2004a, 2004b). Esses trabalhos empregaram as heurísticas SA, BT e AG para resolução de um modelo de regulação florestal inclusões econômicas, e sustentabilidade do empreendimento.

No presente estudo, objetivou-se incluir a disposição espacial das unidades de manejo com intervenções em um modelo de regulação florestal, como fator para um melhor manejo paisagístico e ambiental, bem como a aplicação da meta-heurística algoritmo genético para a resolução do modelo.

\section{MATERIAL E MÉTODOS}

Neste trabalho buscou-se aplicar técnicas alternativas de formulação e inclusão de conceitos ambientais nos modelos de regulação florestal. Para isso, avaliou-se a formulação dos seguintes cenários:

1. minimização das variações das distâncias entre unidades de manejo com intervenção de colheita e o ponto de entrega da produção;

2. maximização do valor presente líquido (VPL), com a aplicação de permissão da variação da distância (5, 10,15 e $20 \%$ ) entre as unidades de manejo com intervenção de colheita e o ponto de entrega da produção;

3. minimização da distância entre as unidades de manejo com intervenções de colheita;

4. maximização do valor presente líquido (VPL), com a aplicação de permissão da variação da distância (5, 10,15 e $20 \%$ ) entre as unidades de manejo com intervenção de colheita.

A avaliação das melhores alternativas de modelagem foi feita em função do VPL e do índice de área de colheita (IAC). Realizou-se a modelagem tradicional, como comparativo econômico, e a aplicação de restrições de adjacência, como valor ambiental, totalizando 10 modelos gerados.

\subsection{Dados}

Os dados utilizados foram obtidos de uma fazenda modelo onde foi implementado o estudo de caso, que representa uma situação encontrada em uma empresa florestal localizada na região centro-leste de Minas Gerais. A área total da fazenda modelo é de aproximadamente 10.000 ha, sendo que $35 \%$ (3.491 ha) são de plantio efetivo e o restante da área constitui-se em reserva legal e áreas de preservação permanente. Ela foi dividida em 135 unidades de manejo em função de características administrativas, edafoclimáticas e fisiográficas.

\subsection{Produção, receita e custo}

Considerou-se somente o plantio de um clone híbrido (Eucalyptus urophylla x Eucalyptus grandis) como sendo a única fonte de madeira utilizada. A curva de produção obtida a partir de dados de parcelas de inventário florestal e utilizada neste estudo foi:

$$
\text { Volume }=\frac{197,833}{1+75,674 e^{-1,315 \text { Idade }}}
$$

em que a idade está em meses.

A tabela de custos empregada foi obtida no trabalho de Binoti (2010), sendo a taxa de juros utilizada de $8,75 \%$ ao ano e o valor de receita com venda da madeira foi de $\mathrm{R} \$ 80,00 / \mathrm{m}^{3}$.

\subsection{Modelos matemáticos}

O modelo foi formulado empregando o sistema RPF (NEUROFOREST, 2012). A abordagem utilizada para a geração de unidades de manejo foi o modelo I proposto por Curtis (1962), com a utilização de variáveis binárias para as variáveis decisórias, resultando em: 
$M A X Z=\sum_{i=1}^{m} \sum_{j=1}^{n} c_{i j} x_{i j}$

sujeito a:

$\sum_{i=1}^{m} \sum_{j=1}^{n} x_{i j}=1$

$\sum_{i=1}^{m} \sum_{j=1}^{n} V_{i j k} x_{i j k} \geq \operatorname{Dmin}_{k} \quad\{k=0,1, \ldots, H-1\}$

$\sum_{i=1}^{m} \sum_{j=1}^{n} V_{i j k} x_{i j k} \leq \operatorname{Dmax}_{k} \quad\{k=0,1, \ldots, H-1\}$

$\sum_{l=1}^{I R} A_{l k}=\left(\frac{\text { Área total }}{I R}\right) \quad\{k=H-1\}$

$x_{i j} \in\{0,1\}$

em que $Z=$ valor presente líquido global (R $\$) ; x_{i j}=$ variáveis de decisão, representando a $j$-ésima alternativa de manejo adotada na $i$-ésima unidade de manejo; $c_{i j}=$ valor presente líquido de cada unidade de manejo $i$, manejada segundo a alternativa de manejo $j ; m=$ número total de unidades de manejo; $n=$ número total de alternativas de manejo na $i$-ésima unidade de manejo; $V_{i j k}=$ volume $\left(\mathrm{m}^{3}\right)$ produzido pela $i$-ésima unidade de manejo quando a $j$-ésima alternativa de manejo é adotada, para o período $k ; \operatorname{Dmin}_{k}$ e $\operatorname{Dmax}_{k}=$ demanda volumétrica $\left(\mathrm{m}^{3}\right)$ mínima e máxima respectivamente, em cada período do horizonte de planejamento; $A_{l k}$ representa a área do povoamento na idade 1 e período $k$; e $I R$ rotação regulatória.

De acordo com esse modelo de programação inteira mista, a maximização do valor presente global (1) está sujeita às restrições de singularidade (2) e (6) e às produções mínimas (3) e máximas (4), em cada período do horizonte de planejamento. A restrição (5) garante o estabelecimento da regulação florestal. Definiu-se o horizonte de planejamento como de 1,5 ciclos, conforme sugerido por Leuschener (1984), sendo a rotação de 6 anos, o horizonte de planejamento foi de 18 anos. As demandas volumétricas anuais máximas e mínimas impostas foram de respectivamente 150.000 e $200.000 \mathrm{~m}^{3}$ em função das necessidades do empreendimento.

As intervenções nos povoamentos incluíram corte seguido de imediata reforma ou corte com reforma no próximo período, o que significa que apenas um regime de manejo (alto-fuste) foi avaliado. Possibilitou-se a variação da idade de corte entre 5 a 9 anos, dentro do horizonte de planejamento, sendo considerada uma rotação regulatória de 6 anos. Para fins de comparação, obteve-se o mesmo modelo sem a inclusão das restrições de adjacência e restrições de classes de idade por compartimento.

O processo de otimização foi realizado pela meta-heurística algoritmo genético (AG). O AG foi implementado em uma rotina computacional, utilizando o ambiente de programação Visual Basic for Applications em conjunto com o Software Microsoft Excel. As soluções (indivíduos) geradas para o problema apresentaram o formato de um vetor $\mathrm{V}(\mathrm{x})=\left\{\mathrm{X}_{11}, \mathrm{X}_{12}, \ldots ., \mathrm{X}_{\mathrm{ij}}\right\}$, onde a variável de decisão $\mathrm{X}_{\mathrm{ij}}\left(\mathrm{X}_{\mathrm{ij}} \in\{0,1\}\right)$, simboliza a alternativa de manejo $\mathrm{j}(\mathrm{j}=1,2, \ldots, \mathrm{n})$ assinalada, ou não, à unidade de manejo i ( $\mathrm{i}=1,2 \ldots \mathrm{m})$ (RODRIGUES et al., 2004a).

A população inicial do $A G$ foi constituída de 30 indivíduos gerados aleatoriamente, considerando a viabilidade de cada solução pela restrição de singularidade. Utilizou-se o crossover de múltiplos pontos e uma taxa de mutação em torno $0,6 \%$ para cada indivíduo da população. A seleção de indivíduos ocorreu por elitização. A seleção dos indivíduos foi feita pelo método da roleta russa. A parada da corrida do algoritmo ocorreu quando, após a estabilização do fitness, o AG realizou 20 novas gerações.

\subsection{Maximização do VPL}

A função de aptidão do algoritmo genético empregada nesse trabalho baseia-se na aplicação de penalidades à função objetivo, que consiste em maximizar o valor presente líquido global. As penalidades impostas referem-se à variação volumétrica acima ou abaixo da demanda proposta, áreas com cortes adjacentes e distribuição irregular de classes de idades ao final do horizonte de planejamento. A função de fitness é apresentada a seguir:

$\operatorname{Max}=\sum_{i=1}^{m} \sum_{j=1}^{n} c_{i j} x_{i j}-\alpha\left(H_{k}-\sum_{i=1}^{m} \sum_{j=1}^{n} V_{i j} x_{i j}\right)^{2}-\beta\left(\sum_{k=0}^{k=h-1} y_{i k}\right)^{2}-\gamma\left(A_{l}-\sum_{i=1}^{m} A_{i} x_{i}\right)^{2}$

em que, $H_{k}=$ demanda volumétrica $\left(\mathrm{m}^{3}\right)$, no $k$-ésimo período do horizonte de planejamento; $\alpha, \beta$ e $\gamma$ são os coeficientes de penalidade associados a cada restrição, valores de penalidades foram de $10^{5} ; y_{i k}$ refere-se ao número de unidades adjacentes cortadas em um mesmo período (as restrições de adjacência empregadas foram do tipo URM - Unit Restriction Model); $A_{l}=$ área regulatória e $A_{i}=$ área da unidade de manejo i.

\subsection{Minimização das variações da distância entre intervenções e o posto de entrega de madeira}

Nesse modelo, buscou-se a minimização das variações ou a manutenção constante da distância entre

Cerne, Lavras, v. 20, n. 1, p. 157-164, jan./mar. 2014 
as intervenções de colheita e a unidade de recepção da produção. A formulação geração da função de aptidão para o AG foi:

Min. $=\sum_{i=1}^{n} D_{i}+\propto\left(H_{k}-\sum_{i=1}^{m} \sum_{j=1}^{n} V_{i j} x_{i j}\right)^{2}+\gamma\left(A_{l}-\sum_{i=1}^{m} A_{i} x_{i}^{\prime}\right.$

em que, $D_{i}$ é a distância real entre a unidade de manejo em situação de colheita e a unidade de recepção de madeira em metros.

2.6 Maximização do VPL com penalidades pela distância entre intervenções e o posto de entrega de madeira

O fitness do algoritmo genético empregado nesse modelo também se baseia na aplicação de penalidades à função objetivo, que consiste em maximizar o valor presente líquido global. As penalidades impostas foram feitas em função de percentagens $(\mu)$ do VPL e da distância entre as unidades de corte e a unidade receptora da produção. As percentagens variaram em progressão aritmética com razão de $5 \%$ tendo valor inicial de $5 \%$ e valor máximo de $20 \%$. A função de aptidão utilizada para esse modelo foi:

$$
\operatorname{Max} .=\sum_{i=1}^{m} \sum_{j j=1}^{n} c_{i j} x_{i j}-\alpha\left(H_{k}-\sum_{i=1}^{m} \sum_{j=1}^{n} V_{i j} V_{i j} x_{i j}\right)^{2}-\mu\left(\sum_{i=1}^{m} \sum_{j=1}^{n} c_{i j} x_{i j}\right)\left(D_{i}\right)-\gamma\left(A_{l}-\sum_{i=1}^{m} A_{i} x_{i}\right)^{2}
$$

\subsection{Maximização das Distâncias entre as Intervenções}

Como alternativa para a aplicação de restrições ambientais e paisagísticas, optou-se pela maximização das distâncias entre as unidades de manejo que estão sofrendo intervenção e a outra unidade de corte mais próxima que também sofre intervenção. A função de aptidão utilizada foi:

$$
\operatorname{Max} .=\sum_{i=1}^{n} d_{i}-\alpha\left(H_{k}-\sum_{i=1}^{m} \sum_{i=1}^{n} V_{i j} x_{i j}\right)^{2}-\gamma\left(A_{l}-\sum_{i=1}^{m} A_{i} x_{i}\right)^{2}
$$

em que, $d_{i}$ representa a distância entre centroides da unidade que está sofrendo intervenção e a outra unidade de corte mais próxima. Foi testada também a manutenção da distância média constante em todos os anos do horizonte de planejamento.

2.8 Maximização do VPL com penalidades pela distância entre unidades de manejo

O fitness do algoritmo genético empregado nesse modelo também se baseia na aplicação de penalidades à função objetivo, que consiste em maximizar o valor presente líquido global. As penalidades impostas foram feitas em função de percentagens $(\mu)$ do VPL e da distância entre as unidades de manejo em colheita e a próxima unidade na mesma situação. As percentagens variaram em razão de 5\% com valor inicial de 5\% e valor máximo de $20 \%$. A função de aptidão utilizada para esse modelo foi:

$\operatorname{Max}=\sum_{i=1}^{m} \sum_{j=1}^{n} c_{i j} x_{i j}-\alpha\left(H_{k}-\sum_{i=1}^{m} \sum_{j=1}^{n} V_{i j} x_{i j}\right)^{2}-\mu\left(\sum_{i=1}^{m} L_{j=1}^{n} c_{i j} c_{i j}\right)\left(d_{i}\right)-\gamma\left(A_{l}-\sum_{i=1}^{m} A_{i} x_{i}\right)^{2}$

\subsection{Restrição de Adjacência}

Para comparação, construiu-se um modelo com a aplicação clássica de restrições de adjacência. Pela topografia muito acidentada da área de estudo, as unidades de manejo foram consideradas adjacentes quando a distância entre seus limites (bordas) era menor ou igual a $50 \mathrm{~m}$. A restrição de adjacência para evitar o corte de unidades de manejo adjacentes em cada período $\mathrm{k}$ foi:

$$
n_{i} x_{i j}+\sum_{i \in N_{i}} x_{i j} \leq n_{i} \forall i
$$

em que $n_{i}$ refere-se ao número de unidades de manejo adjacentes que apresentam cortes no período $\mathrm{k} \mathrm{e} \mathrm{N}_{\mathrm{i}}$ refere-se ao conjunto de unidades de manejo adjacentes à i-ésima unidade de manejo considerada (MCDILL; BRAZE, 2000).

\subsection{Avaliação da Dispersão das Intervenções nas Unidades de Manejo}

Entre as diversas métricas utilizadas para a avaliação do manejo da paisagem encontradas na literatura (BASKENT; JORDAN, 1995), optou-se por avaliar o efeito da restrição de adjacência, pela média ponderada do inverso da menor distância ao quadrado entre unidades de manejo cortadas no mesmo período, pelas áreas ao quadrado das unidades de manejo que sofrem intervenção. O índice utilizado foi:

$I A C=\frac{\sum_{k=0}^{H-1} \sum_{i=1}^{m} A_{i}^{2}\left(\frac{1}{d_{i}}\right)^{2}}{\sum_{i=1}^{m} A_{i}}$

em que, $\mathrm{IAC}=$ índice de área de colheita, $\mathrm{A}_{\mathrm{i}}=$ área da unidade de manejo i com intervenção, $\mathrm{em} \mathrm{m}^{2}, \mathrm{~d}_{\mathrm{i}}=$ distância até a unidade de manejo mais próxima com intervenção, em metros.

Cerne, Lavras, v. 20, n. 1, p. 157-164, jan./mar. 2014 


\section{RESULTADOS}

A formulação do modelo de planejamento resultou em um modelo com 8.755 variáveis decisórias. As formulações das restrições de adjacência resultaram em 37.555 restrições. Todos os modelos testados supriram a demanda volumétrica imposta. A imposição das restrições de adjacência reduziu o VPL global em torno de $8 \%$. O valor da função objetivo para os modelos com e sem a inclusão das restrições de adjacência foi de $\mathrm{R} \$ 17.143 .857,17$ e $\mathrm{R} \$ 18.599 .169,37$, respectivamente.

O valor do IAC para o modelo com e sem a inclusão da restrição de adjacência foi de 0,246436 e 0,615032, respectivamente. O modelo com a manutenção das distâncias entre as unidades de manejo com intervenções de colheita e a unidade de entrega da produção apresentou uma redução de menos de $1 \%$ em relação ao modelo com restrições de adjacência, sendo que o valor do IAC para esse modelo foi de 0,243089 . A variação do VPL e do IAC referentes aos demais modelos que consideram a distância entre as unidades de manejo com corte e a unidade de entrega da produção é apresentada nas Figuras 1 e 2.

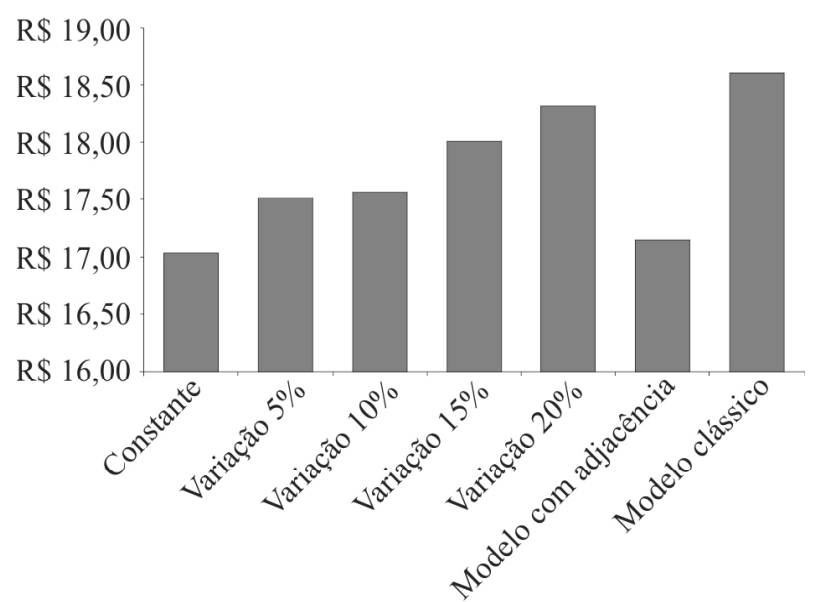

Figura 1 - Valores de VPL, em milhões de reais, referentes às distâncias entre as unidades de manejo com corte e a unidade de entrega da produção.

Figure 1 - Values of NPV in millions of dollars, referring to the distances between the management units with cutting and the delivery unit of production.

Os modelos com a relação às distâncias entre as unidades de manejo que sofreram intervenções não apresentaram soluções viáveis, contudo as restrições volumétricas e regulatórias foram satisfeitas. O modelo

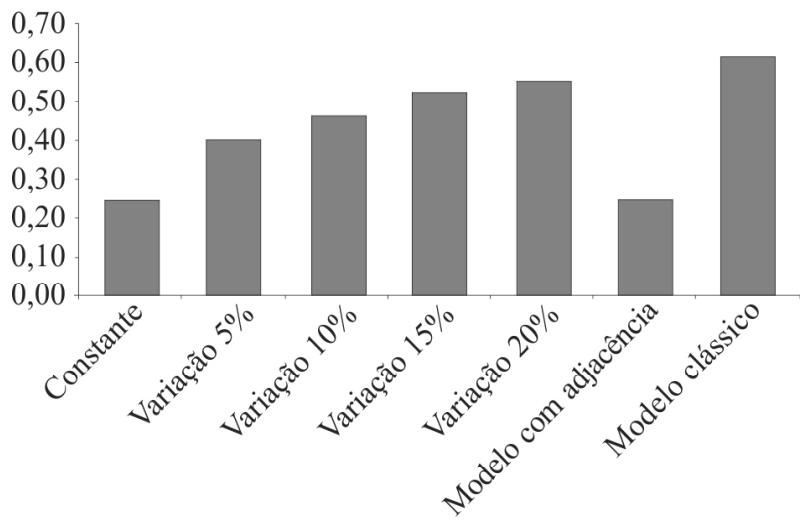

Figura 2 - Valores de IAC referentes às distâncias entre as unidades de manejo com corte e a unidade de entrega da produção.

Figure 2-Values of IAC concerning the distances between the management units with cutting and the delivery unit of production.

com a maximização das distâncias entre as unidades de manejo que sofreram intervenções apresentou um aumento de $1 \%$ com relação ao valor do modelo com adjacência, entretanto, o valor para o IAC foi de 0,326927 . O modelo que objetivou a redução da variação da distância entre as unidades de manejo que sofreram intervenções, apresentou uma melhoria no valor do IAC com relação ao modelo com adjacência e uma pequena variação com relação ao VPL. A comparação dos resultados de VPL e IAC, entre os modelos que consideração as variações entre as distâncias entre as unidades de manejo é apresentada nas Figuras 3 e 4 .

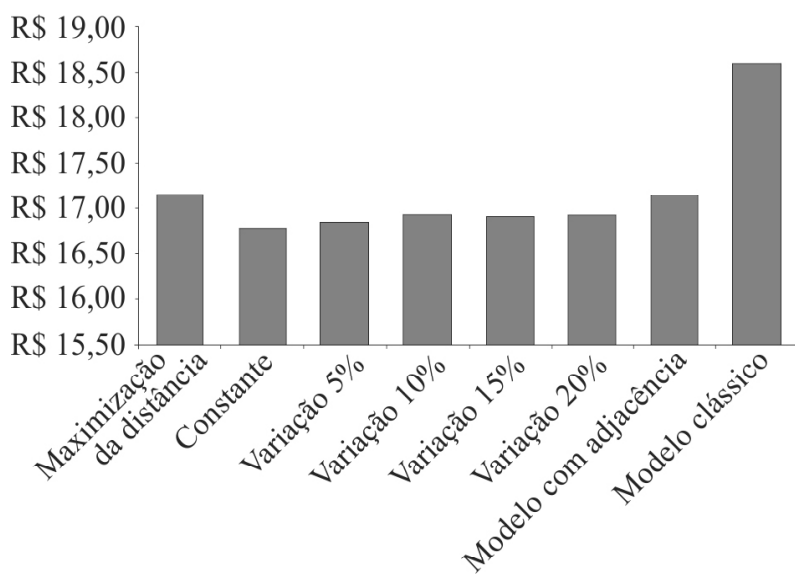

Figura 3 - Valores de VPL, em milhões de reais, referentes às distâncias entre as unidades de manejo com corte.

Figure 3 - Values of NPV in millions of reais, referring to the distances between the management units with harvest.

Cerne, Lavras, v. 20, n. 1, p. 157-164, jan./mar. 2014 


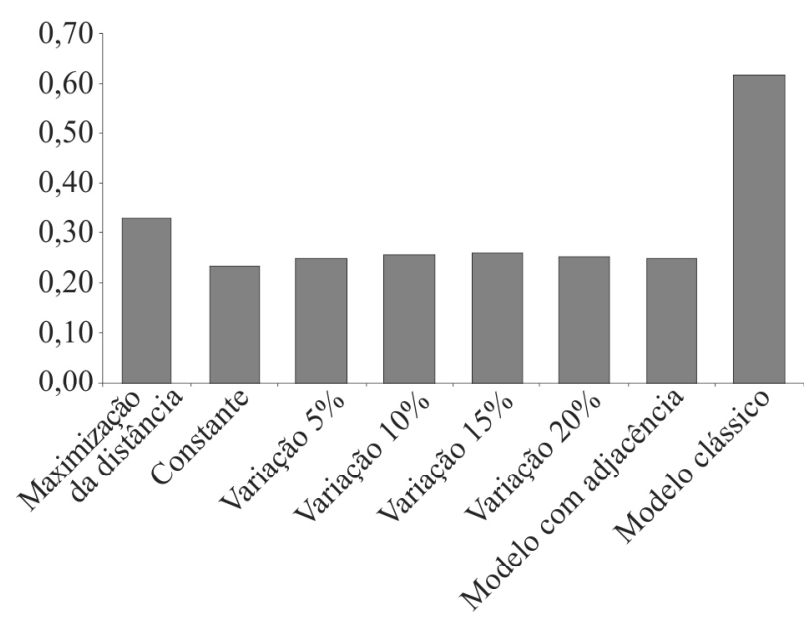

Figura 4 - Valores de IAC referentes às distancias entre as unidades de manejo com corte.

Figure $4-I A C$ relating to the distance between management units with harvest.

\section{DISCUSSÃO}

A paisagem florestal é formada por um mosaico de unidades de manejo distintas que se interagem funcionalmente (TURNER, 1989). A interação entre as unidades de manejo decresce drasticamente com a distância (STRANGE et al., 2002). Neste estudo, são apresentadas alternativas para a inclusão das distâncias entre unidades de manejo em um modelo de regulação florestal.

A principal forma de aplicação de restrições ambientais nos modelos de regulação florestal consiste na aplicação de restrições de adjacência (CASTRO, 2007; MOREIRA, 2008). Uma dificuldade da implementação dessas restrições, consiste na dimensão que o modelo de regulação assume, exigindo um enorme esforço computacional (BASKENT; JORDAN, 2005). Outra desvantagem na utilização dessas restrições consiste na complexidade adicionada ao problema, muitas vezes, não apresentando soluções factíveis (CASTRO, 2007).

O índice utilizado para a comparação dos modelos apresenta-se extremamente simples e de fácil obtenção com a aplicação de técnicas de Sistemas de Informações Geográficas (SIG) ou cartográficas. O índice apresentase consistente com base nos pressupostos adotados para o manejo de paisagem de plantios equiâneos. O índice é diretamente proporcional à área que sofre intervenção e inversamente proporcional à distância entre as unidades de manejo com intervenção, ponderada pela área de corte anual. Em termos práticos, quanto mais se aproximar de zero, melhores serão as influências ambientais e socioeconômicas sobre os ecossistemas e comunidades circunvizinhas.

A minimização da variação da distância entre as unidades de manejo em situação de corte e o posto de entrega da madeira, tende a dispersar a colheita, distribuindo esta por toda a floresta. Essa forma de modelagem apresentou-se satisfatória para a solução do problema tratado nesse estudo, podendo substituir a modelagem com restrições de adjacência. A aplicação de penalidades ao fitness em função das variações das restrições apresenta uma melhoria econômica com relação ao modelo de adjacência, contudo o beneficio ambiental é reduzido.

A maximização das distâncias entre as unidades de manejo com intervenção apresentou uma melhoria no valor do IAC e uma redução do VPL, quando comparado com o modelo clássico. Os demais modelos que consideraram a manutenção da distância entre as unidades de manejo constante, ou permitindo variações, não apresentaram soluções satisfatórias para essas restrições, ou seja, o resultado da função objetivo apresenta reduções em função das penalidades pelo fato de essas soluções não suprirem as necessidades destas restrições. Contudo, a solução obtida com a aplicação desses modelos apresentou valores de IAC e VPL similares ao modelo com restrições de adjacência, sendo esta uma alternativa promissora para a inclusão de características ambientais em modelos de regulação florestal.

\section{REFERÊNCIAS}

AARTS, E.; LENSTRA, J. K. Introduction. In: Local search in combinatorial optimization. $\mathrm{New}$ York: J. Wiley, 1997. p. 116.

BARE, B. B.; MENDOZA, G. A. Timber harvest scheduling in a fuzzy decision environment. Canadian Journal of Forest Research, Ottawa, v. 22, p. 423-428, 1992.

BASKENT, E. Z.; JORDAN, G. A. Characterising spatial structure of forest landscape. Canadian Journal of Forest Research, Ottawa, v. 25, p. 18301849, 1995.

BASKENT, E. Z.; JORDAN, G. A. Forest landscape management modelling using simulated annealing.

Forest Ecology and Management, Amsterdam, v. 165, p. 2945, 2002. 
BASKENT, E. Z.; JORDAN, G. A. Spatial wood supply simulation modelling. Forestry Chronicle, Ottawa, v. 67, n. 6, p. 610-621, 1991.

BASKENT, E. Z.; KELES, S. Spatial forest planning: a review. Ecological Modelling, Amsterdam, v. 188, p. 145-173, 2005.

BETTINGER, P.; BOSTON, K.; KIM, Y. H.; ZHU, J. Landscape-level optimization using tabu search and stand density-related forest management prescriptions. European Journal of Operational Research, Amsterdam, v. 176, p. 1265-1282, 2007.

BETTINGER, P.; BOSTON, K.; SIRY, L. P.; GREBNER, R. L. Forest management and planning. San Diego: Elsevier, 2009. 331 p.

BETTINGER, P.; GRAETZ, D.; BOSTON, K.; SESSIONS, J.; CHUNG, W. Eight heuristic planning techniques applied to three increasingly diffcult wildlife planning problems. Silva Fennica, Helsinski, v. 36, p. 561-584, 2002.

BETTINGER, P.; SESSIONS, J.; BOSTON, K. Using tabu search to schedule timber harvests subject to spatial wildlife goals for big game. Ecological Modelling, Amsterdam, v. 42, p. 111123, 1997.

BINOTI, D. H. B. Estratégias de regulação de florestas equiâneas com vistas ao manejo da paisagem. 2010. 157 f. Dissertação (Mestrado em Ciência Florestal) - Universidade Federal de Viçosa, Viçosa, 2010.

CASTRO, R. R. Regulação de florestas equiâneas incluindo restrições de adjacência. 2007. 64 f. Dissertação (Mestrado em Ciência Florestal) Universidade Federal de Viçosa, Viçosa, 2007.

CHURCH, R. L.; MURRAY, A. T.; WEINTRAUB, A. Locational issues in forest management. Location Science, Chicago, v. 6, p. 137-153, 1998.

CURTIS, F. H. Linear programming the management of a forest property. Journal of Forestry, Washington, v. 9, p. 611-616, 1962.
HEINONEN, T. Developing spatial optimization in forest planning. 2007. $34 \mathrm{f}$. Dissertation (Master in Forestales) - University of Joensuu, Joensuu, 2007.

HILLER, F. S.; LIEBERMAN, G. J. Introdução à pesquisa operacional. 8. ed. Rio de Janeiro: McGrawHill, 2010. 828 p.

LEUSCHNER, W. A. Introduction to forest resource management. New York: J. Wiley, 1984. 284 p.

LOCKWOOD, C.; MOORE, T. Harvest scheduling with spatial constraints: a simulated annealing approach. Canadian Journal of Forest Research, Ottawa, v. 23, p. $468475,1993$.

MCDILL, M. E.; BRAZE, J. Comparing adjacency constraint formulations for randomly generated forest planning problems with four age-class distributions. Forest Science, Bethesda, v. 46, p. 423-436, 2000.

MOREIRA, J. M. M. A. P. A incorporação de corredores de conectividade em problemas de otimização para o planejamento de florestas industriais. 2008. $123 \mathrm{f}$. Tese (Doutorado em Economia Aplicada) - Escola Superior de Agricultura "Luiz de Queiroz", Piracicaba, 2008.

MURRAY, A.; CHURCH, R. Heuristic solution approaches to operational forest planning problems. Operation Research Spektrum, Berlin, v. 17, p. 193203, 1995.

NEUROFOREST. Disponível em: $<$ http://neuroforest. ucoz.com>. Acesso em: 10 jun. 2012.

ÖHMAN, K.; ERIKSSON, L. O. Allowing for spatial consideration in longterm forest planning by linking linear programming with simulated annealing. Forest Ecology and Management, Amsterdam, v. 161, p. 221230, 2002.

PUKKALA, T.; KURTTILA, M. Examining the performance of six heuristic search techniques in different forest planning problems. Silva Fennica, Helsinski, v. 39, n. 1, p. 6780, 2005.

RICHARDS, E. W.; GUNN, E. A. Tabu search design for difficult forest management optimization problems.

Cerne, Lavras, v. 20, n. 1, p. 157-164, jan./mar. 2014 
Canadian Journal of Forest Research, Ottawa, v. 33, n. 6, p. 11261133, 2003.

RODRIGUES, F. L. Metaheurística e sistema de suporte à decisão no gerenciamento de recursos florestais. 2001. 225 f. Tese (Doutorado em Ciência Florestal) - Universidade Federal de Viçosa, Vicosa, 2001.

RODRIGUES, F. L.; LEITE, H. G.; SANTOS, H. N.; SOUZA, A. L. Soluções de problemas de planejamento florestal com restrições de inteireza utilizando busca tabu. Revista Árvore, Viçosa, v. 27, n. 5, p. 701-713, set./out. 2003.

RODRIGUES, F. L.; LEITE, H. G.; SANTOS, H. N.; SOUZA, A. L.; RIBEIRO, C. A. A. S. Metaheurística simulated annealing para solução de problemas de planejamento florestal com restrições de integridade. Revista Árvore, Viçosa, v. 28, n. 2, p. 247-256, mar./ abr. 2004a.

RODRIGUES, F. L.; LEITE, H. G.; SANTOS, H. N.; SOUZA, A. L.; SILVA, G. F. Metaheuristica algoritmo genetic para solução de problemas de planejamento florestal com restrições de integridade. Revista Árvore, Viçosa, v. 22, n. 2, p. 233-245, mar./abr. 2004 b.

STRANGE, N.; MEILBY, H.; THORSEN, J. T. Optimizing land use in afforestation areas using evolutionary self-organization. Forest Science, Bethesda, v. 48, p. 543-555, 2002.

TURNER, M. G. Landscape ecology: the effect of pattern on process. Annual Review of Ecology and Systematics, Palo Alto, v. 20, p. 171-197, 1989.

WANG, Y.; ZHANG, X. A dynamic modeling approach to simulating socioeconomic effects on landscape changes. Ecological Modelling, Amsterdam, v. 140, p. 141-162, 2001.

Recebido: 14 de abril de 2011; aceito: 25 de junho de 2013.

Cerne, Lavras, v. 20, n. 1, p. 157-164, jan./mar. 2014 